\title{
BSL2 Audit and Certification Program: An Effort to Harmonize and to Raise Standards in Both Laboratory Infrastructure and Biosafety Practices in Singapore
}

\author{
Tin Tun ${ }^{\dagger}$ and Lee Kien Wah \\ Nanyang Technological University, Office of Health and Safety (OHS), 637551, Singapore
}

\begin{abstract}
The critical aspects of biosafety and bio-containment have been increasingly important in recent years. Biological agents involved in biological research projects at the Nanyang Technological University (NTU) Singapore are usually those with low risks. Biosafety level 2 or BSL 2 laboratories are widely used. However, biosafety measures which refer to the implementation of laboratory practices and procedures, specific construction features of laboratory facilities and safety equipment must be in place to reduce the exposure of laboratory personnel, the public or the environment to potentially infectious agents or other biological hazards. It is also required to pay more attention to laboratory-acquired infections (LAIs) which may occur in research laboratories, clinical laboratories or animal facilities. BSL 2 audit and certification program is implemented as an internal exercise covering laboratories in the university where biological agents are handled or biological research works are carried out. We have put some efforts to raise biosafety standards university-wide in both laboratory infrastructure and laboratory practices to a higher level. Common audit findings are briefly discussed in this presentation.
\end{abstract}

Key Words: BSL 2 laboratories; Laboratory audit; Laboratory certification; Biosafety competency

\section{INTRODUCTION}

The Ministry of Health $(\mathrm{MOH})$ in Singapore regulates the use of high risk biological agents1 to comply with Biological Agents and Toxins Act (BATA) (Singapore Statutes Online. Biological Agents and Toxins Act. 2005). A biosafety level 3 or BSL 3 facility is required to handle risk group 3 biological agent or more aptly known as BATA Schedule 1 (part 2) biological agent (Ministry of Health of Singapore. Updated Biological Agents and Toxins List. 2016). The Act requires every facility operator to appoint an Institutional Biosafety Committee (IBC) which formulates biosafety policies and control measures for safe functioning of the facility. In Singapore, it is a requirement for a BSL 3 laboratory to be inspected yearly, certified and registered with the $\mathrm{MOH}$ as a certified facility.

The $\mathrm{MOH}$ allows appeal for use of some high risk biological agents in a BSL 2 laboratory with BSL 3 practices. A special permit is issued specifying certain instructions or requirements and the laboratory is registered as an uncertified or BSL $2+$ facility. The IBC needs to submit annual report to the $\mathrm{MOH}$ for those BSL 3 and BSL 2+ facilities. Genetic manipulation work or project involving use of genetically modified organism (GMO) needs to seek review and approval from the Genetic Modification Advisory Committee

\footnotetext{
*Received: August 21, 2016 / Revised: September 29, 2016 / Accepted: September 29, 2016

${ }^{\dagger}$ Corresponding author: Tin Tun. Nanyang Technological University, Office of Health and Safety (OHS), 637551, Singapore.

Tel: +65-6136-7898, Fax: +65-65-6592-7556, e-mail: ttin@ntu.edu.sg

(C) The Korean Society for Biomedical Laboratory Sciences. All rights reserved.

(c) This is an Open Access article distributed under the terms of the Creative Commons Attribution Non-Commercial License (http://creativecommons.org/licenses/by-nc/3.0/) which permits unrestricted non-commercial use, distribution, and reproduction in any medium, provided the original work is properly cited.
} 
(GMAC). For research compliance, the committee develops the Singapore Biosafety Guidelines for Research on Genetically Modified Organisms (GMAC. Singapore Guidelines for Research on GMOs. 2001).

BSL 2 laboratories are allowed to use for biological projects involving biological agents with low risk groups (Risk Groups 1 and 2 or biological agents described in Schedule 4 of BATA) and GMO. The operations and control used in BSL2 laboratories are generally under the control of the Ministry of Manpower (MOM) under the general ambit of the Workplace Safety and Health Act (WSHA) (MOM, Singapore. Workplace Safety and Health Act. 2006). But these BSL 2 laboratories are yet to be legally certified or registered in Singapore. It is known that the $\mathrm{MOH}$ and/or the MOM are looking into biosafety issues for BSL 2 laboratories but no specific requirements have been defined so far. However, these research laboratories involve not only biological risk; there are other associated risks such as radiation, chemical, physical and mechanical nature. Biological risk is generally more complicated to handle as micro-organisms exhibit pathogenic and virulence characteristics. Thus this audit and certification program is unique and an internal exercise to bring the NTU biosafety up to a higher level and more importantly, to discharge our legal duties as doing what is reasonably practicable under the WSHA.

\section{NTU BSL 2 LABORATORIES}

The University comprises of many schools and research centers. There is an Animal Research Facility on campus for research use. All together 133 laboratories from seven schools and three research centers were included in this program. Some schools - namely School of Biological Sciences, School of Chemical and Biomedical Engineering and Lee Kong Chian School of Medicine - play very important roles for fundamental biological and biomedical research. Two research centers - Nanyang Environment and Water Research Institute and Singapore Centre on Environmental Life Sciences Engineering - carry out many projects involving biological agents for both fundamental and applied research.

\section{BIOSAFETY ISSUES}

Under the University's Health Safety and Environment (HSE) Steering Committee, the IBC looks into biosafety issues and advises the University on all biological and related matters. The Office of Health and Safety (OHS) plays the role of IBC Secretariat. The IBC and the OHS conduct audits, routine inspections and unscheduled checks for any biological work to ensure all the risks are adequately addressed and additionally seeks to assure the senior management that all foreseeable risks are taken under control. All biological project proposals are reviewed - by the IBC and registered with the Biological Project Number (BPN) on the online application system. BPN Administrators are appointed at every school or research center to monitor biological projects and to facilitate the administrative process between the researchers and IBC on biosafety issues.

\section{PROGRAM OBJECTIVES}

As a start, there is a need to do gap analysis of the requirements of good biological research laboratories and the developed institutional standard for both facilities requirement and practices.

This program (gap analysis) was implemented to fulfil the following objectives.

1. To access the adequacy of laboratory facilities and equipment;

2. To ensure biosafety measures and good microbiological practices (GMP) are implemented for storage, safe handling and disposal of biological agents, toxins and GMO and bio-materials (cells, samples, reagents);

3. To form a database of all BSL 2 laboratories in NTU schools and centers where research works involving biological agents or GMO are handled; and

4. To ensure all projects handling biological agent or GMO have the required $\mathrm{BPN}$ registration.

The gaps noted in the audit of laboratories provide each lab with the continual improvement targets to ensure that the safety of the workers and also the integrity of the biological samples handled. 
Table 1. A simplified checklist for audit purpose was prepared for biosafety requirements based on WHO Biosafety Manual, the NTU Safety Manual for Biological Work and local requirements

\section{CHECKLIST FOR BSL 2 LABORATORIES}

Lab Location:

PI / In-charge: Inspected by:

Biosafety Level (BSL):
School / Research centre:

BPN Administrator:

Person(s) interviewed:

Date:

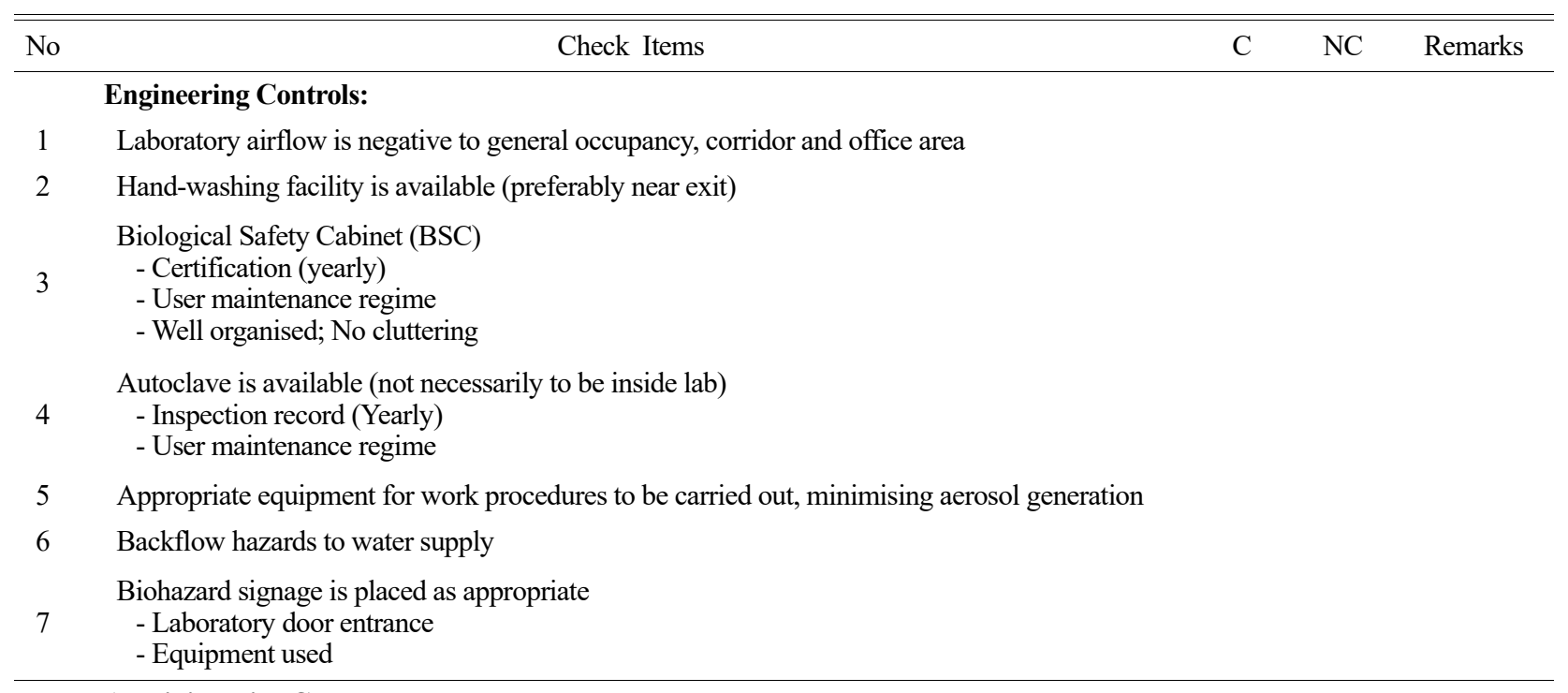

\section{Administrative Controls:}

1 Access control (restricted to authorized personnel)

2 All contaminated waste materials are autoclaved or decontaminated chemically before disposal

3 Biological wastes are decontaminated and stored in properly labelled containers (including sharps)

4 Biological agent in possession are properly packed and labelled with agent name, storage conditions and user name

$5 \quad$ Flammables are stored in approved cabinet

$6 \quad$ Chemicals are properly stored, used and disposed

7 Risk assessment and Hazard communication with lab users

\section{Personal Protection:}

1 Use of personal protective equipment

1 .lab coat; gloves; covered shoes; eye, face, respiratory protection

2 Laboratory coats, gloves and PPE are not worn outside the lab

3 Personal protective equipment for cryogenic transfer (gloves)

$4 \quad$ PPE are stored in proper place after use

5 Health surveillance and disease monitoring of staff (pre-employment health check

5 for BSL 2 staff) + Immunisation Records
Emergency Response Procedure:
1 Biological and Chemical Spill Kits are available and easily accessible
2 Laboratory personnel trained in emergency response
3 First-aid kit and OFAs are available 
Table 1. A simplified checklist for audit purpose was prepared for biosafety requirements based on WHO Biosafety Manual, the NTU Safety Manual for Biological Work and local requirements (Continued)

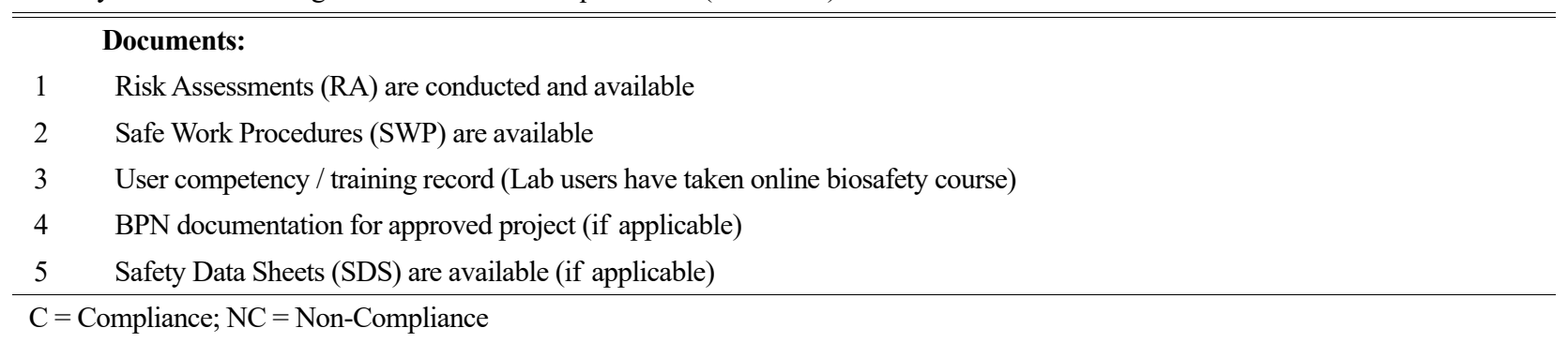

\section{LABORATORY AUDIT}

Laboratory audit was scheduled from August 2015 to January 2016 for the NTU laboratories where biological agents and genetically modified organisms (GMO) are handled. Audit was conducted under guidance of the IBC and close supervision of the Chief Health and Safety Officer (CHSO). A simplified checklist for audit purpose was prepared for biosafety requirements based on WHO Biosafety Manual (WHO. Laboratory biosafety manual. 2004) the NTU Safety Manual for Biological Work and local requirements (Table 1). Audit methodology include review of documents (including all important work and biological risk assessments), interview of laboratory users, physical assessment or inspection and audit report and follow up actions. Safety Officers from the OHS participated in the physical inspection of laboratories and documentation of findings. BPN administrators, principal investigators and researchers or laboratory users in NTU schools or centers assisted and cooperated very well. Follow up and corrective actions upon audit findings were taken accordingly.

\section{COMMON AUDIT FINDINGS AND COMMENTS}

Audit was carried out using the simplified checklist. Common findings and some areas which need safety improvements were summarized in Table 2 and Fig. 1. General comments on findings were made at least to meet minimum requirements for biosafety measures which are: facility requirements for a basic biological research (ventilation and security); adequacy of commonly used equipment and their maintenance regime; good microbiological practices (GMP); and laboratory users' competency in biosafety practices and knowledge. The University has a set of online biosafety training which workers should have completed and passed prior to commence research work in the laboratory.

\section{Basic requirements for a facility}

Laboratory design and room pressure: Many laboratories showed positive or no difference in room pressure to common corridors or outside areas (74.4\%; 99 out of total 133). Several of those laboratories were set up originally not for biological experiment or designed with "Clean Room" concept and air blower entry and had a positive room pressure. This differential ventilation concept is important because the inward negative pressure serves to contain any airborne aerosols within the laboratory thus minimizing contamination of the external environment and reducing risks. Pressure differentials across laboratory doorways should be negative to general occupancy, corridor and office area so that all possible biological risks will be contained inside the laboratory. Very high differential also indicates substantial ingress of unconditioned air in the laboratory and may result in high cooling cost. Adjustment of room pressure and regular checking were advised.

Hand wash facility: A designated hand washing sink was not available in some laboratories $(16.5 \%$; 22 out of 133) (Fig. 2). There are facilities comprising a large main laboratory made up of many small isolated rooms equipped with biological safety cabinet for purpose of handling biological agent. But there were no hand wash sink available nearby 
Table 2. 133 BSL 2 laboratories from 7 schools or 3 research centers were audited. There are many areas where some safety requirements or safety gaps were identified. Laboratories are to make improvements in those areas and to take corrective actions upon findings

\begin{tabular}{|c|c|}
\hline Area of audit findings & $\begin{array}{l}\text { No. of labs } \\
\text { (out of 133) }\end{array}$ \\
\hline $\begin{array}{l}\text { Laboratories showed positive or no difference in room pressure to general occupancy common corridor or outside area. } \\
\text { Fail to contain all possible biological risks inside the laboratory. Regular check and Adjustment will be necessary. }\end{array}$ & $\begin{array}{c}99 \\
(74.4 \%)\end{array}$ \\
\hline Designated sink for hand washing is not available; no proper signage or no provision of detergent or drying facility & $\begin{array}{c}22 \\
(16.5 \%)\end{array}$ \\
\hline $\begin{array}{l}\text { Poor maintenance for centrifuge. SOP for safe operation; emergency procedure; responsibility and maintenance records } \\
\text { are not available. }\end{array}$ & $\begin{array}{c}3 \\
(2.3 \%)\end{array}$ \\
\hline $\begin{array}{l}\text { Improper use of BSC; clutters inside the BSC during operation; use of BSC as a storage facilities by keeping laboratory } \\
\text { apparatus and stuff not required for experiment; no clean, dirty and working zones being practiced }\end{array}$ & $\begin{array}{c}56 \\
(42.1 \%)\end{array}$ \\
\hline $\begin{array}{l}\text { Poor maintenance of autoclave corroded with dirty water inside; procedures detailing responsibilities, proper maintenance } \\
\text { and cleaning of autoclave not available. }\end{array}$ & $\begin{array}{c}36 \\
(27.1 \%)\end{array}$ \\
\hline $\begin{array}{l}\text { Incomplete laboratory documentation; standard operating procedures (SOP) and safe work procedures (SWP), equipment } \\
\text { maintenance records, inventory of biological agents, training record, accident and incident record, etc. are not available. }\end{array}$ & $\begin{array}{c}54 \\
(40.6 \%)\end{array}$ \\
\hline $\begin{array}{l}\text { RA documents do not address many activities with significant risks; control measures are not in place; no records for } \\
\text { communication with lab users }\end{array}$ & $\begin{array}{c}21 \\
(15.8 \%)\end{array}$ \\
\hline $\begin{array}{l}\text { Disposal of biological waste without prior autoclaving, inactivation or decontamination before leaving the laboratory } \\
\text { or the facility }\end{array}$ & $\begin{array}{c}37 \\
(27.8 \%)\end{array}$ \\
\hline $\begin{array}{l}\text { Disposal of liquid biological waste into lab sink after disinfecting with chlorine or other disinfectants. Toxic industrial } \\
\text { waste (TIW) are not to discard to sewage. }\end{array}$ & $\begin{array}{c}38 \\
(28.5 \%)\end{array}$ \\
\hline $\begin{array}{l}\text { Lack of proper hand over or take over practice; when a project has ended or a researcher leaves, all associated biological } \\
\text { samples or stuff shall either be transferred to another researcher or inactivated and disposed of. }\end{array}$ & $\begin{array}{c}3 \\
(2.3 \%)\end{array}$ \\
\hline
\end{tabular}

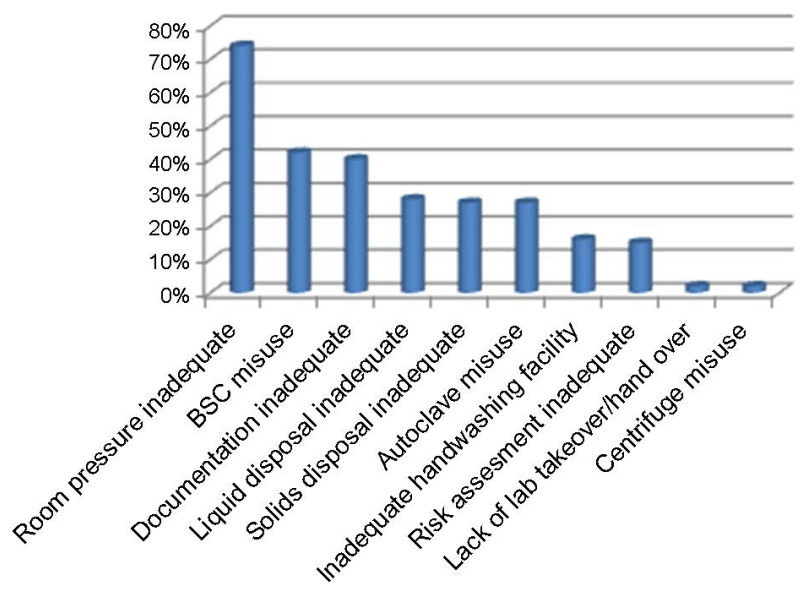

Fig. 1. A graphical presentation of summarized audit findings.

those small rooms. Laboratory should have a designated sink for hand washing. The hand washing sink is to be free of any chemical or biological substances to minimize contamination. Sink for individual lab may sometimes not be possible; in which case, a shared hand washing sink for the cluster of labs can be considered, preferably located near

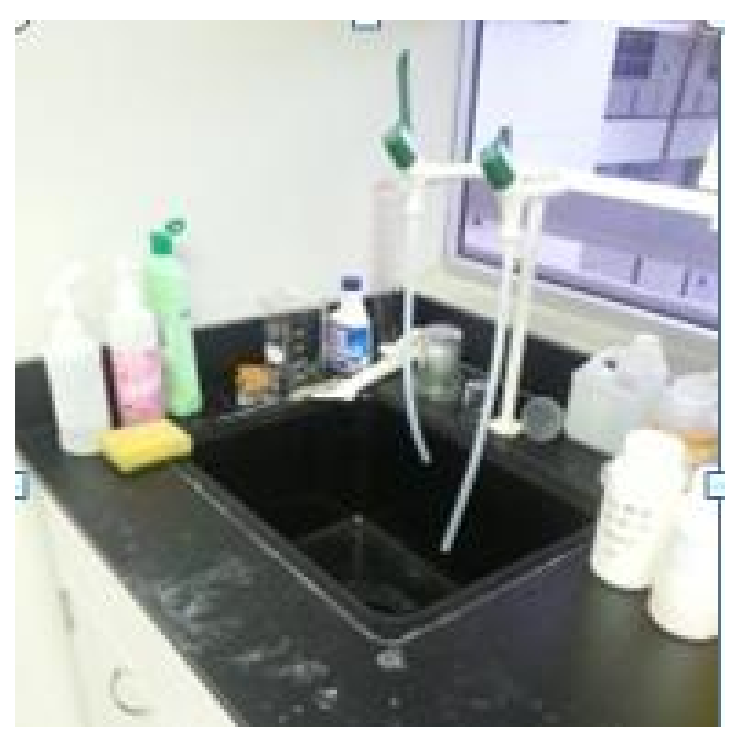

Fig. 2. No designated hand wash sink is available. No proper signage or no provision of detergent or hand drying facility. Laboratory users use laboratory sink for hand wash.

the exit door. A label "For Hand Washing Only" should be posted on the top to remind lab users. Such sink should 

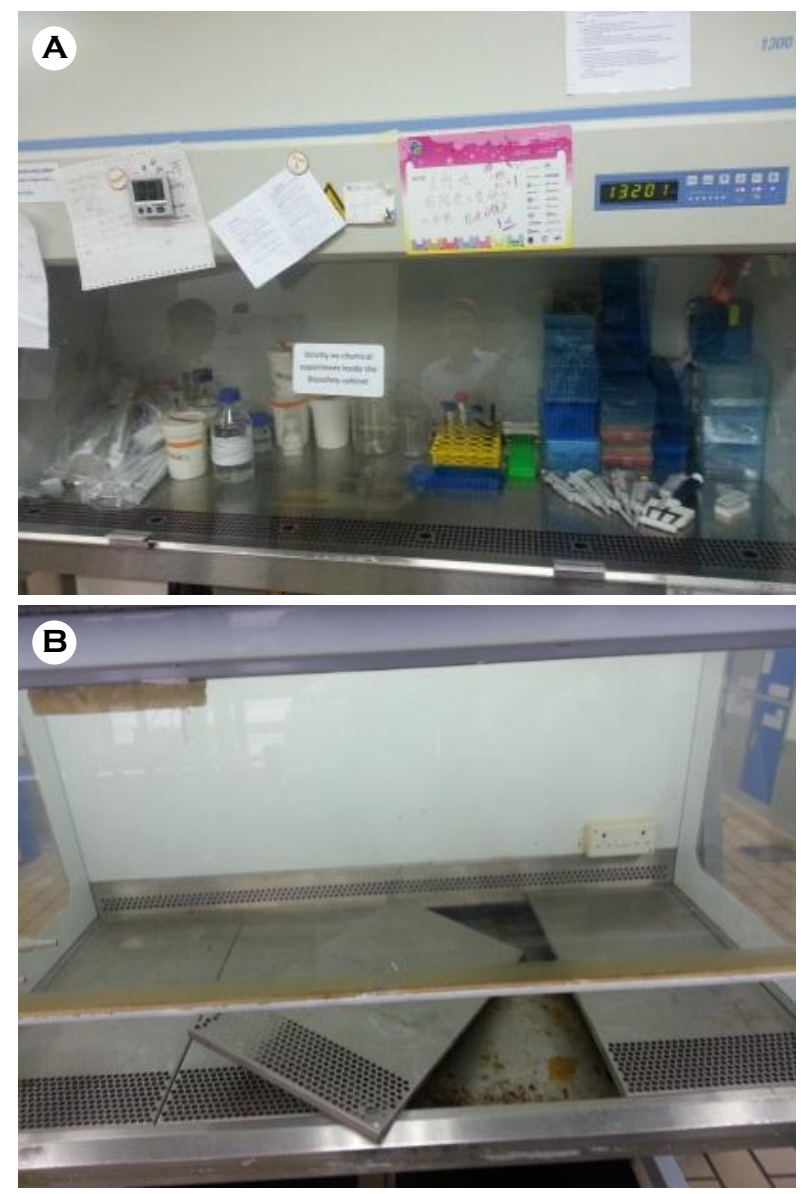

Fig. 3. The BSC is too crowded; clutters inside; keeping things not required for experiment and use the BSC for storage (A). No proper maintenance and cleaning after use (B).

also be washed and sanitized frequently due to possible residual microorganisms. Provision of hand detergent and hand drying facilities should be considered.

\section{Common laboratory equipment in a biological labora- tory}

Biological Safety Cabinet (BSC): The BSC must be duly tested and certified annually by competent person with regard to air flow and HEPA filter to meet acceptable standards or manufacturer's specifications. The BSC shall be properly maintained and kept clean, sanitized, well organized and free of clutter. This should be done after every use, if not at the end of the day. Any blockage within the BSC can cause dis turbance of airflow and reducing the effectiveness of the
BSC in protecting the users from the biological agent. Nothing should be left inside after use. It is therefore vital that these rules must be adhered. Unfortunately, improper use of BSC was observed in many laboratories (42.1\%; 56 out of 133). Laboratory users do not understand proper use of BSC such as blocking the inlet grills, and are not aware of the avoidance of clutters inside the BSC during operation. It was noted that many used BSC as a storage facilities by keeping laboratory apparatus and stuff not required for experiment. There were no clean, dirty and working zones being practiced (Fig. 3).

Autoclave: It was found that a legislation requirement has been duly met since autoclaves (pressured vessels) in all schools or centers bore MOM issued inspection certificates. Despite the mandatory inspection, poor maintenance of autoclave corroded with dirty water inside was observed (Fig. 4A). An operating procedure detailing responsibilities, proper maintenance and cleaning of autoclave should be in place, especially so if the autoclave is a shared facility by many different users.

Centrifuge: The centrifuge should be properly maintained and kept clean all times. It was noted that some centrifuges were lack of proper maintenance (Fig. 4B). Equipment needs a clean-up after use and regular check conducted or service regime implemented for proper functioning. Careful balancing of rotor buckets and safety locking system are very important for safe use of equipment to prevent high speed aerosol generation. Loading and unloading of buckets with biological samples should be done inside the BSC. Transportation of loaded centrifuge bucket should be carried out with care using secondary containment and trolley as required.

\section{Good Microbiological Practices (GMP)}

Laboratory coat: It was noted in the audit that some laboratory only impose wearing of laboratory coat only when user conducts an experiment. Person not doing laboratory work or visitor including contractor is allowed to enter the laboratory without wearing a laboratory coat and moving close to the biological work activities, ignoring the possi- 

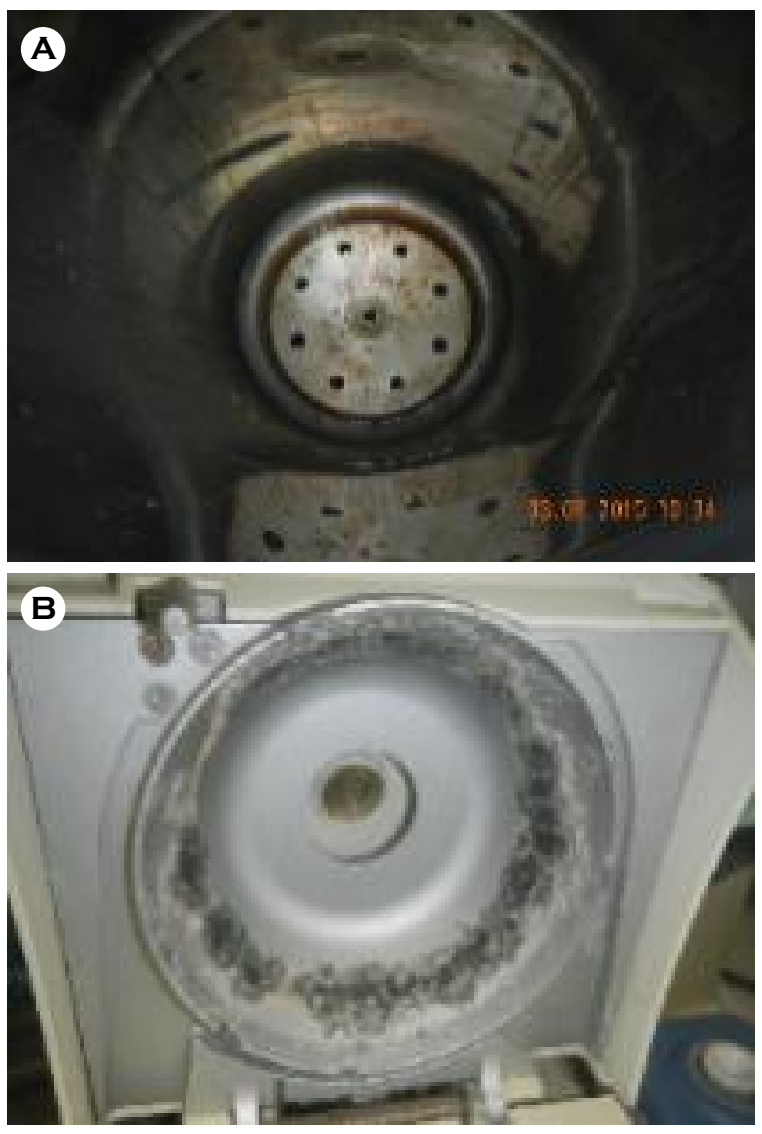

Fig. 4. Poor maintenance of equipment: (A) corroded autoclave with dirty water inside. (B) Corroded and dirty centrifuge cover.

bility of surface contamination. It is for this reason that all should wear laboratory coat inside the laboratory. More importantly, in a shared laboratory, someone may be working and no one should be allowed to enter to any bench working space without wearing a laboratory coat. As to common corridor area for thoroughfare (if so determined by the safety committee after risk assessment) may be demarcated clearly by lines for visitors and research staff alike without the use of laboratory coat. There is a need to review laboratory coat policy.

Use of personal protective equipment: Personal protective equipment for example, goggle, gloves and face shield or face mask are used for additional controls against contact risks with the biological agents and other physical hazards. It was noted that some researchers used liquid nitrogen for

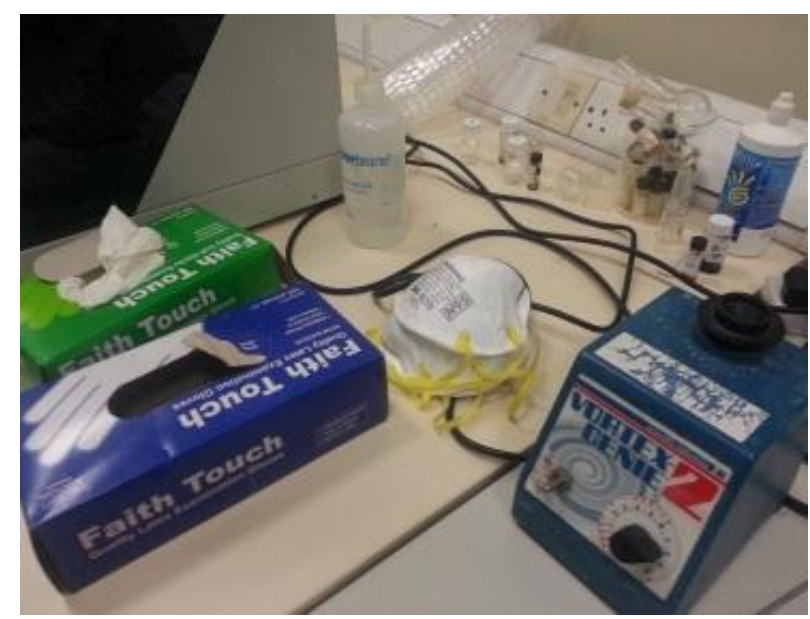

Fig. 5. Used N95 mask was left on the bench together with other contaminated items. It should be disposed of immediately after its use. In case of reusable mask, the mask should be sanitized and sealed in plastic bag and kept away separately.

storage of biological items without applying cryogenic gloves or face shield. It was also observed that used N95 mask was left on the bench together with other contaminated items (Fig. 5). It is very important not to touch inner part of the N95 mask and used one should be disposed of immediately after its use. In case of reusable mask, the mask should be sanitized and sealed in plastic bag and kept away separately. Proper use of personal protective equipment must be practiced.

Bio-waste disposal: It was observed that solid biological wastes are collected by the bio-waste contractor without autoclaving or inactivation, departing from required practice under the NTU biological manual (27.8\%; 37 out of 133$)$. This is not a good practice as it can cause laboratory acquired infection (LAI) especially during transportation and also possibility of third party (such as a waste collection worker or members of the public) being infected. It is advised that all bio-waste should be autoclaved, inactivated or decontaminated before leaving the laboratory and/or the facility. Storage of contaminated biologically active wastes with other chemical wastes also increased the risk of contamination and propagation resulting in widespread infection to non-suspecting persons. Bio-waste should be autoclaved 


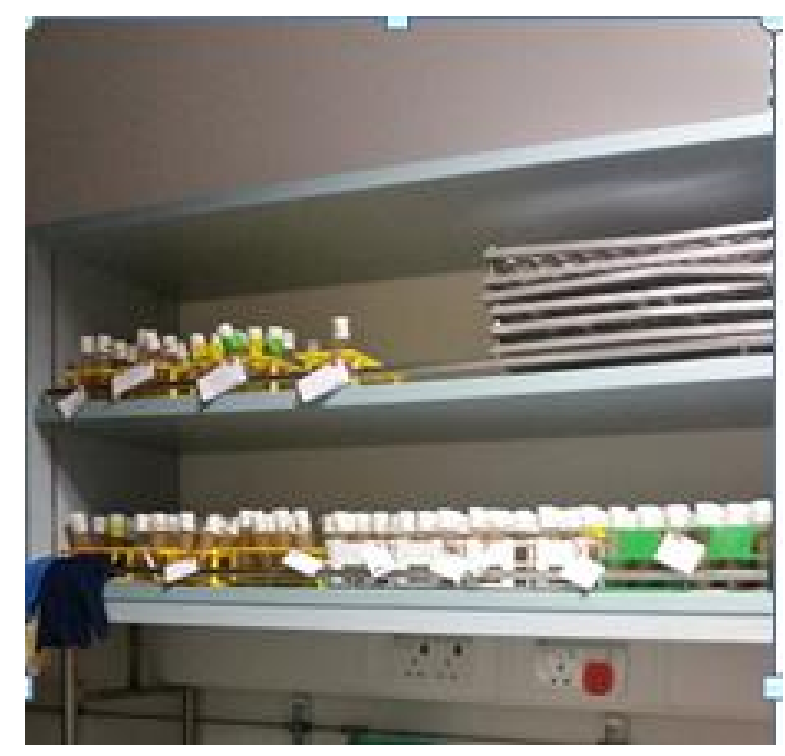

Fig. 6. When a project has ended or a researcher leaves, all associated biological stock and retained samples shall either be transferred to another researcher or inactivated and disposed of. The lack of such practices results in accumulation of remaining bacterial cultures, stocks and reagents that no one is aware of.

or decontaminated before leaving the laboratory. It is surprising that we note that some researchers disposed of liquid biological waste into the sink after inactivation $(28.5 \%, 38$ out of 133). The inclusion of chemical disinfectant and cause disruption in the waste treatment plant and should be discouraged and in certain chemical disinfectant pose risks to sewerage plant workers.

Hand over clearance: When a project has ended or a researcher leaves, all associated biological stock and retained samples shall either be transferred to another researcher or inactivated and disposed of. The lack of such documented procedure can result in accumulation of remaining bacterial cultures, stocks and reagents that no one is aware of (Fig. 6). It is necessary to develop and practice hand over and take over procedure when a principal investigator, researcher or student leaves such that all biological agents are properly accounted for and adequately dealt with.

Laboratory documentation: It is necessary to keep laboratory documentation in order and make it available. Some documents such as risk assessment (RA) and safety data sheet (SDS) are legally required. Other important documents to keep are standard operating procedures (SOP) and safe work procedures (SWP), equipment maintenance records, inventories of biological agents in possession, training records, accident and incident records, etc. In general, laboratory documents were available but there was a need to improve quality of documentation in many laboratories (40.6\%; 54 out of 133). Some examples were that RA does not address many activities with significant risks; equipment maintenance records are not systematically updated; operating procedures for equipment are not available; biological agents in possession are not recorded.

\section{User's competency in biosafety}

User's competency in biosafety is very important for safe handling of biological agents and biological materials which can cause laboratory acquired infection (LAI). Laboratory user must have appropriate biosafety training which includes biosafety concept, legislation requirements, good microbiological practices (GMP) and emergency procedures and/or spill management. Online Basic Biosafety training program is available for the NTU laboratory users and all workers in biological laboratories are expected to have completed the course and the tests before being allowed into the facility.

The development of such basic competency in biological and biomedical workers is the basis of the introduction of a national biosafety training program, known as the Biosafety Competency Framework, by WDA (Work Development Agency) with the strong endorsement of $\mathrm{MOM}$ and $\mathrm{MOH}$ is in process. The strength of such program will be standardized or harmonized practices in this area and promote mutual recognition of competency of laboratory users from various institutions - hospitals, universities, polytechnics, research centers and other industries. When the program is ready and publicized, employers have to send their staff to the agency-approved training provider or training institute. "Biosafety Passport" will be awarded upon completion of the course. Holder of the passport will be well qualified and recognized as a competent worker in any biological laboratory in Singapore. 


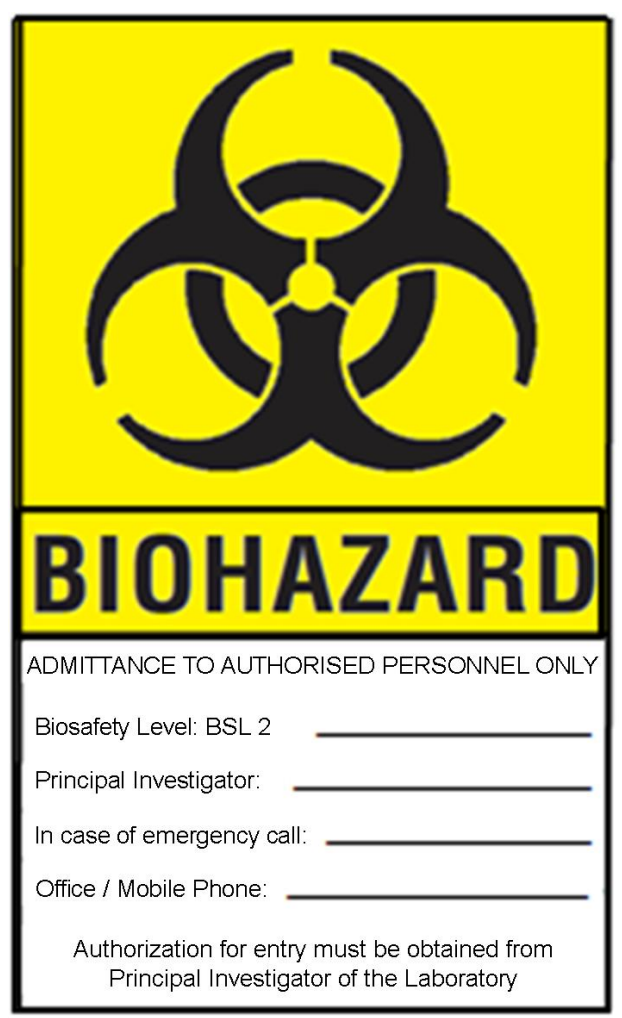

Fig. 7. Standardized biohazard signage is posted at the doors of the certified BSL 2 laboratory.

\section{LABORATORY CERTIFICATION}

The "BSL 2 Certification Criteria" document was developed for strictly internal use to identify requirements which defines a minimum standard in safe control of biological risks in a BSL 2 laboratory in the NTU. The document is condensed from recommendations laid down in WHO Biosafety Manual, BMBL from CDC [6] and legal requirements in Singapore. This document is available for the NTU laboratory users as a shared document on the OHS webpage. Laboratory certification is the systematic review of all safety features and processes associated with the laboratory. This include assessment of a) engineering controls such as biosafety cabinets and ventilation; b) administrative control such as work risk assessments, biological risk review, standard operating procedures (SOP), documentation and record retention systems; and c) use of personal protective equipment. Having met the requirements and set criteria, the inspected or audited NTU laboratories are registered as BSL 2 laboratories. A standardized biosafety signage will be posted at the doors of all certified laboratories (Fig. 7).

\section{FUTURE BIOSAFETY IMPROVEMENTS}

There is a need to raise commitment to biosafety for laboratory users at multiple levels. Biosafety training, registration of certified NTU BSL 2 laboratories, biological project registration (BPN) process, biosafety measures and practices are important areas which demand continuous improvements. It will be also required to get the "NTU Safety Manual for Biological Work" reviewed and updated with information based on important outcomes of this program. Review of current online biosafety course which is an internal training for NTU laboratory users, may be needed to ensure that it can be a useful platform for integration to future national "Biosafety Passport" program. Above all, there is a constant need to ensure good practices are implemented with the principal investigators and lab managers being key drivers in this process.

\section{CONCLUSIONS}

Basic equipment required for biological research works are provided at NTU laboratories. Laboratory facility is mostly adequate and sufficient for safe handling of biological agent with lower risk provided that proper maintenance of equipment and good microbiological practices are in place. As laboratories, including NTU's labs, are to move forward and handle more novel, innovative and perhaps more virulence substances, there is a constant need to train our researchers and to mold the new safety culture in biological and biomedical research to be vigilant and adhere to good microbiological practices.

Commitment toward biosafety has been inconsistent and insufficient at multiple levels. Individual schools/centers, teams and research groups have taken it upon themselves to implement biosafety practices, but this is not done in a consistent manner. Integrated safety culture and a standardized biosafety practice needs to be established. This is an expectation of the program and all persons from the top 
down are accountable.

\section{Abbreviations}

BSL, Biosafety Level; NTU, Nanyang Technological University; $\mathrm{MOH}$, Ministry of Health; MOM, Ministry of Manpower; IBC, Institutional Biosafety Committee; BATA, Biological Agents and Toxins Act; WSHA, Workplace Safety and Health Act; GMO, Genetically Modified Organism; GMAC, Genetic Modification Advisory Committee; GMP, Good Microbiological Practices; BSC, Biological Safety Cabinet; BPN, Biological Project Number; RA, Risk Assessment; SDS, Safety Data Sheet; LAI, Laboratory Acquired Infection

\section{Authors' contributions}

All authors contributed equally to this work.

\section{Authors' declarations}

This work used data obtained from the laboratory audit program report. Laboratory audit and certification program was conducted as an internal exercise for university. Mr Tin Tun, who is the Programme Manager and first author, prepared this manuscript and Dr Lee Kien Wah, who is the Chief Safety and Health Officer and co-author, approved the manuscript. Authors take responsibility for all ethical issues.

\section{Acknowledgements}

Sincere appreciations go to IBC members for their guidance and support; Safety Officers (OHS) for participation in site audit inspection and assessment of audit findings; BPN Administrators for their assistance in audit inspection of laboratories at schools and research centers; and, Laboratory users or Researchers for their cooperation in this audit and certification program.

\section{Conflict of interest}

The authors declare that they have no competing interests.

\section{REFERENCES}

Available at: http://www.mom.gov.sg/legislation/workplace-safetyand-health

Available at: http://www.who.int/csr/resources/publications/biosafety /Biosafety7.pdf

Available at: http://www.gmac.gov.sg/Index_Singapore_Biosafety_ Guidelines_for_Research_on_GMOs.html

Available at: http://statutes.agc.gov.sg/aol/search/display/view.w3p; page $=0$; query $=$ DocId\%3A6b6eae33-48b3-4aeb-bbbd-651b1 4629c01\%20Depth\%3A0\%20ValidTime\%3A01\%2F10\%2F 2011\%20TransactionTime\%3A22\%2F09\%2F2011\%20Statu $\mathrm{s} \% 3$ Ainforce;rec $=0$; whole $=$ yes\#P1I-

Available at: https://www.moh.gov.sg/content/dam/moh_web/ BioSafety/docs/List_of_Biological_Agents_and_Toxins.pdf

Centers for Disease Control and Prevention (CDC) National Institute of Health (NIH). Biosafety in Microbiological and Biomedical Laboratories (5rd ed). 2009.

Genetic Modification Advisory Committee (GMAC). Singapore Guidelines for Research on GMOs. 2001

Ministry of Health of Singapore. Updated Biological Agents and Toxins List. 2016

Ministry of Manpower (MOM), Singapore. Workplace Safety and Health Act. 2006.

World Health Organization (WHO). Laboratory biosafety manual (3rd ed). 2004

Singapore Statutes Online. Biological Agents and Toxins Act. 2005. 